\title{
Study Day of Morphology Assessment
}

National Cancer Institute

\section{Source}

National Cancer Institute. Study Day of Morphology Assessment. NCI Thesaurus. Code C117630.

The day that a morphology assessment is performed. 